\title{
The perception of visually presented yaw and pitch turns: Assessing the contribution of motion, static, and cognitive cues
}

\author{
MANUEL VIDAL \\ Laboratoire de Physiologie de la Perception et de l'Action, \\ CNRS/Collège de France, Paris, France \\ MICHEL-ANGE AMORIM \\ Université Paris Sud, Orsay, France \\ and \\ JOSEPH MCINTYRE and ALAIN BERTHOZ \\ Laboratoire de Physiologie de la Perception et de l'Action, \\ CNRS/Collège de France, Paris, France
}

\begin{abstract}
Terrestrial gravity restricts human locomotion to surfaces in which turns involve rotations around the body axis. Because observers are usually upright, one might expect the effects of gravity to induce differences in the processing of vertical versus horizontal turns. Subjects observed visual scenes of bending tunnels, either statically or dynamically, as if they were moving passively through the visual scene and were then asked to reproduce the turn deviation of the tunnel with a trackball. In order to disentangle inertia-related (earth-centered) from vision-related (body-centered) factors, the subjects were either upright or lying on their right side during the observations. Furthermore, the availability of continuous optic flow, geometrical cues, and eye movement were manipulated in three experiments. The results allowed us to characterize the factors' contributions as follows. Forward turns (pitch down) with all cues were largely overestimated, as compared with backward turns (pitch up). First, eye movements known to be irregular for vertical stimulation were largely responsible for this asymmetry. Second, geometry-based estimations are, to some extent, asymmetrical. Third, a cognitive effect corresponding to the evaluation of navigability for upward and downward turns was found (i.e., topdown influences, such as the fear of falling often reported), which tended to increase the estimation of turns in the direction of gravity.
\end{abstract}

The updating of one's perceived orientation when one is turning around the body axis in the horizontal plane (yaw rotations) can be performed either by extracting one's heading from optic flow when visual cues are present (Bertin, Israël, \& Lappe, 2000; Lappe, Bremmer, \& van den Berg, 1999; Warren \& Hannon, 1988; Warren, Kay, Zosh, Duchon, \& Sahuc, 2001) or by integrating vestibular acceleration (Israël, Bronstein, Kanayama, Faldon, \& Gresty, 1996; Israël, Sievering, \& Koenig, 1995; Ivanenko, Grasso, Israël, \& Berthoz, 1997) and proprioceptive cues (Amorim, Glasauer, Corpinot, \& Berthoz, 1997; Mittelstaedt, 1999). All these studies showed a sym-

This research was supported by the Centre National d'Études Spatiales (CNES). M.V. received a grant from the Centre National de Recherche Scientifique (CNRS) for his Ph.D. The authors thank Michaël Zugaro and Sid Wiener for their helpful comments and all the subjects who kindly participated in the experiments. Correspondence concerning this article should be addressed to M. Vidal, LPCMV, TCR AVA 013,1 avenue de Golf, F-78288 Guyancourt cedex, France (e-mail: manuel.vidal@lpcmv .cnrs.fr). metrical perception of turnings, whether the subjects were going leftward or rightward.

Less is known about the perception of pitch rotations performed around a horizontal axis. Cohen and Larson (1974) asked subjects to adjust the pitch of their bodies in complete darkness to 13 different goal orientations between prone and supine positions. They found that the subjects underestimated their pitch orientation when they were tilted less than $60^{\circ}$ backward or forward from the vertical and overestimated their pitch orientation when they were nearly prone, indicating an asymmetry in the processing of vestibular and somatosensory cues in order to estimate their body pitch orientation. A strong asymmetry in pitch-induced sensations was also reported when a pitch-rotating optic flow subtending a large field of view was observed (Young, Oman, \& Dichgans, 1975). Downward pitch stimuli generated a significantly stronger pitch sensation than did upward pitch stimuli. This asymmetry in the vertical plane turns (pitch) was absent in horizontal plane turns (yaw).

It is likely that the difference in gravity's effects on pitch versus yaw rotations can explain the differences in behav- 
ior between these two actions, at both a physiological and a cognitive level. Indeed, there are several anatomical and physiological characteristics of the central nervous system relating to gravity that might come into play. First of all, pitch and yaw rotations stimulate physiological sensors in a fundamentally different fashion. In natural conditions, humans stabilize their heads in an upright posture (Pozzo, Berthoz, \& Lefort, 1990); therefore, the gravity axis matches the rotation axis for yaw rotations. Under these circumstances, the receptors sensing gravity are essentially unperturbed, whether one is turning rightward or leftward. This is not so for pitch rotations, in which the otoliths of the inner ear and other organs sensitive to gravity can potentially sense the absolute orientation of the head and body with respect to the vertical. The normal Earth environment also differentiates between pitch and yaw turns with respect to what such an environment affords to the individual. ${ }^{1}$ The act of turning the body left or right in an upright position does not have the same consequences for the animal as that of pitching forward or backward.

Humans, like every living species on earth, have evolved in the presence of gravity, which may have led to a nervous system organization that distinguishes the processing of up/down and left/right directions (Young, 1984). Indeed, the head direction cell system of rats does not supply information on the pitch component of the direction taken by the animal's head (Stackman, Tullman, \& Taube, 2000). In weightlessness, the horizontal plane associated with these head direction cells is projected onto the surface on which the animal is walking (Knierim, McNaughton, \& Poe, 2000; Taube, Muller, \& Ranck, 1990). These findings suggest an internal organization of space that processes distinctly spatial information relative to elevation and azimuth. Furthermore, gravity certainly had an influence on the evolution of the human cognitive mechanisms involved in spatial orientation. Both the behavioral requirements of navigating in an essentially 2-D world and the constraints of remaining upright with respect to gravity would strongly influence the evolution of perceptual and behavioral mechanisms. We would expect this phylogenetic functional adaptation to result in a different processing of upward and downward turns, independently of the actual sensing of gravity.

\section{Experiment Rationale}

In the present investigation, we measured human subjects' perception of pitch and yaw turns presented visually while they remained in a fixed position. The subjects observed a visual display of optic flow consistent with a movement through a virtual, curved corridor. The task for the subjects was to indicate the perceived angle of the bend. According to what Loomis and Beall (1998) have suggested for the control of locomotion, we supposed that when the subjects traveled inside our corridors, the extraction of the deviation angle of the turn would result from the processing of three types of information: (1) motion cues during turns, provided by optic flow and implicit body rotations; (2) static cues, such as the 3-D shape of corridors; and (3) cognitive cues concerning scene af- fordances for these turns. It is important to note that the vestibular information provided during the virtual motion in these experiments was not realistic. Indeed, during the visual turns, there was no angular acceleration or gravity orientation change that could be sensed by the semicircular canals or the otoliths, respectively. By establishing sensory conflict between graviceptor information and the visual or cognitive cues listed above, we set out to test how these different factors interact to provide a composite perception of the body's orientation in 3-D space.

To this end, we conducted three experiments in which we manipulated the sensory information available to the subject when he or she was traversing the curved virtual corridors. The primary experimental manipulation was to test the subject's perception in an upright and reclined position. By manipulating the subject's body position, we were able to examine the respective contribution of factors defined in the visual body-centric reference frame and of factors defined in the inertial earth-centric reference frame in this perceptual process (see the right panel of Figure 2). Once the subject was in the reclined position, the factors generating an asymmetry in the perception of turns would either be tilted together with the subject if they were defined in a body-centric reference frame or would remain aligned with earth vertical if they were defined in an earth-centric reference frame. This technique of dissociating the usually aligned gravity and body reference frames had also been applied in a previous investigation in which we studied the memorization process of a complex path (Vidal, Amorim, \& Berthoz, 2004; Vidal \& Berthoz, 2005). We then further refined the sensory cues available to the subject in the viewing of the virtual movement. In Experiment 1, eye movements were not constrained during the stimulation. Thus, both static and motion visual cues were available. In Experiment 2, the same simulation of a continuous motion was used, but this time the subject had to keep his or her gaze fixed. Since he or she could not scan the visual scene to extract relevant geometrical information, cues, such as slant orientation, that are available only in the periphery of the visual field were not available. Thus, in Experiment 2, only visual motion cues could be processed to estimate the turn angle. In Experiment 3, the subject simply observed static images of the same turning corridors as seen from the viewpoint just before the turn. This last experiment was run in order to study the effect of gravity when the subject was simply processing the 3-D geometrical cues provided by static visual scenes.

Table 1 summarizes the list of potential factors and mechanisms that could have an asymmetrical influence on the perception of turns, classified in two groups: those related to gravity, hence defined in an earth-centric reference frame, and those related to vision and, thus, defined in an egocentric reference frame. In the right section of this table, we specify, for each experiment, in which condition of body orientation (upright or lying down) they might have an influence. This table provides the logical basis for the results of each of the three experiments performed here and will be referred to extensively throughout the discussions of the results. 
Table 1

Potential Factors and Mechanisms That Might Have an Asymmetrical Influence on the Perception of Turns, Classified According to the Reference Frame in Which They Are Defined (e.g., Egocentric or Earth-Centric) and the Kind of Cue Processed (Motion, Static, or Cognitive)

\begin{tabular}{|c|c|c|c|c|c|c|c|c|c|c|c|}
\hline \multirow[b]{2}{*}{ Factor } & \multirow[b]{2}{*}{$\begin{array}{c}\text { Asymmetrical } \\
\text { Factor Description }\end{array}$} & \multirow[b]{2}{*}{$\begin{array}{l}\text { Processed } \\
\text { Cues }\end{array}$} & \multicolumn{3}{|c|}{ Experiment 1} & \multicolumn{3}{|c|}{ Experiment 2} & \multicolumn{3}{|c|}{ Experiment 3} \\
\hline & & & $\begin{array}{l}\text { Pitch } \\
\text { UP }\end{array}$ & $\begin{array}{l}\text { Pitch } \\
\text { LD }\end{array}$ & $\begin{array}{l}\text { Yaw } \\
\text { LD }\end{array}$ & $\begin{array}{l}\text { Pitch } \\
\text { UP }\end{array}$ & $\begin{array}{l}\text { Pitch } \\
\text { LD }\end{array}$ & $\begin{array}{l}\text { Yaw } \\
\text { LD }\end{array}$ & $\begin{array}{l}\text { Pitch } \\
\text { UP }\end{array}$ & $\begin{array}{l}\text { Pitch } \\
\text { LD }\end{array}$ & $\begin{array}{l}\text { Yaw } \\
\text { LD }\end{array}$ \\
\hline \multicolumn{12}{|c|}{ Inertial related (earth-centric reference frame) } \\
\hline 1 & $\begin{array}{l}\text { Input of gravity in the path } \\
\text { integration mechanisms } \\
\text { (vestibular and somatosensory) }\end{array}$ & Motion cues & $\mathrm{X}$ & & $\mathrm{X}$ & $\mathrm{X}$ & & $\mathrm{X}$ & & & \\
\hline 2 & $\begin{array}{l}\text { Scene navigability deriving from } \\
\text { the orientation of gravity }\end{array}$ & Cognitive cues & $\mathrm{X}$ & & $\mathrm{X}$ & $\mathrm{X}$ & & $\mathrm{X}$ & $\mathrm{X}$ & & $\mathrm{X}$ \\
\hline \multicolumn{12}{|c|}{ Vision related (egocentric reference frame) } \\
\hline 3 & $\begin{array}{l}\text { Input of optic flow in the path } \\
\text { integration mechanisms (vision) }\end{array}$ & Motion cues & $\mathrm{X}$ & $\mathrm{X}$ & & $\mathrm{X}$ & $\mathrm{X}$ & & & & \\
\hline 4 & $\begin{array}{l}\text { 3-D geometrical processing of } \\
\text { the environment }\end{array}$ & Static cues & $\mathrm{X}$ & $\mathrm{X}$ & & & & & $\mathrm{X}$ & $\mathrm{X}$ & \\
\hline 5 & $\begin{array}{l}\text { Scene navigability deriving from } \\
\text { the visual orientation }\end{array}$ & Cognitive cues & $X$ & $\mathrm{X}$ & & $\mathrm{X}$ & $\mathrm{X}$ & & $\mathrm{X}$ & $\mathrm{X}$ & \\
\hline
\end{tabular}

Note-For each experiment, we specified in which condition of body orientation — upright (UP) or lying down (LD) — these factors might have an influence.

\section{EXPERIMENT 1 Motion and Static Cues for Turn Perception}

In this experiment, we tested the ability of human subjects to perceive the angle of turns in visually presented stimuli that consisted of passively driven virtual movements (optic flow) inside corridors with a bend. We first tested the subjects in an upright, seated position-looking for differences in the perception of turns with or against gravity - or in the horizontal plane. We then tested the subjects when they were lying on their right side. In this position, we anticipated that either pitch or yaw turns could produce asymmetric error responses with regard to turn direction, each one resulting from effects in the processing of egocentric and gravicentric perceptual cues defined in the visual and inertial reference frames, respectively.

\section{Method}

Subjects. Sixteen naive subjects ( 12 men and 4 women), between 20 and 32 years of age, participated in this experiment; most were students or laboratory staff, and all but one were right-handed. All gave prior written consent before starting this investigation.

Experimental setup. The subjects faced a large screen, either sitting on a chair of adjustable height or lying on a bed on their side in a $90^{\circ}$ roll position. In both situations, the line of sight was centered on the large screen subtending $97^{\circ}$ of horizontal and vertical field of view (FOV), onto which the images of virtual movements were projected (apparatus detailed in Figure 1). The lights in the room were turned off in order to avoid any external visual disturbance.

Procedure. Each trial in the experiment included a visual navigation phase followed by a reproduction task. During the navigation phase, the subjects were exposed to a simulation of passive selfmotion, which corresponded to what they would have seen if they had moved inside of a bending corridor. The tangential speed was kept constant during the whole displacement. The corridors with stone-textured walls were in the form of a pipe with constant circular cross-sections, and each contained a single turn in the middle (see Figure 2A). Each turn could be either in pitch or in yaw ${ }^{2}$ (as defined in Figure 1), with 12 deviation angles that ranged from $\pm 45^{\circ}$ to $\pm 157.5^{\circ}$ in increments of $22.5^{\circ}$. Just after the navigation phase, the subjects were asked to reproduce with a trackball the angle of the curve within the corridor. They could see a picture of a flexible tube representing an outside view of the corridor. The tube was viewed from the top for yaw turns or from the side for pitch turns (see Figure $2 \mathrm{~B})$. The corridor was initially presented as a straight tube $\left(0^{\circ}\right.$ deviation), and the subjects were instructed to bend the tube to the desired turn amplitude, rolling the ball of the trackball horizontally for yaw turns or vertically for pitch turns. They pressed the right button to indicate when they had reproduced the deviation angle corresponding to the angle perceived during the navigation. The next trial followed automatically after $5 \mathrm{sec}$.

The full experiment for a single subject comprised two sessions of 96 trials each, divided into four uninterrupted blocks of 24 trials. A block included 12 pitch turn trials followed by 12 yaw turn trials, or vice versa. Each of the 12 signed angles of turn (leftward and rightward or forward and backward) occurred just once and in a random order for every half-block. Therefore, each turn was executed four times for all the crossed conditions (body position $\times$ turn orientation $\times$ turn direction $\times$ turn deviation angle). At the end of each block, feedback about the subject's performance was displayed before a short pause. This feedback was the error (in degrees) measured between the real experienced turn angle and the reproduced one, averaged over all trials in the block. The subject's body position (upright or lying on the right side) was changed after two blocks of trials, and the initial body position was counterbalanced across subjects.

The experiment was preceded by four practice trials with the subjects in the seated position: two trials with pitch turns and two trials with yaw turns. During these trials, the subjects learned how to use the computer interface. The full experiment lasted approximately $90 \mathrm{~min}$ : $50 \mathrm{~min}$ for the first session, which included the instructions and practice trials, and $40 \mathrm{~min}$ for the second session. The subjects were requested to rest for at least $1 \mathrm{~h}$ between sessions.

Realism of the virtual motion. During displacement through the virtual corridor, gaze direction rotated in anticipation of the curve, as would be done in natural conditions (Grasso, Glasauer, Takei, \& Berthoz, 1996; Wann \& Swapp, 2000). That is, the virtual viewing direction started rotating $1,150 \mathrm{msec}$ before the translation of the viewpoint started to curve. This anticipation delay was estimated empirically: We tested different delays and chose the one that was the most natural and comfortable. Linear speed was kept constant during the whole displacement and was the same for every trial and 

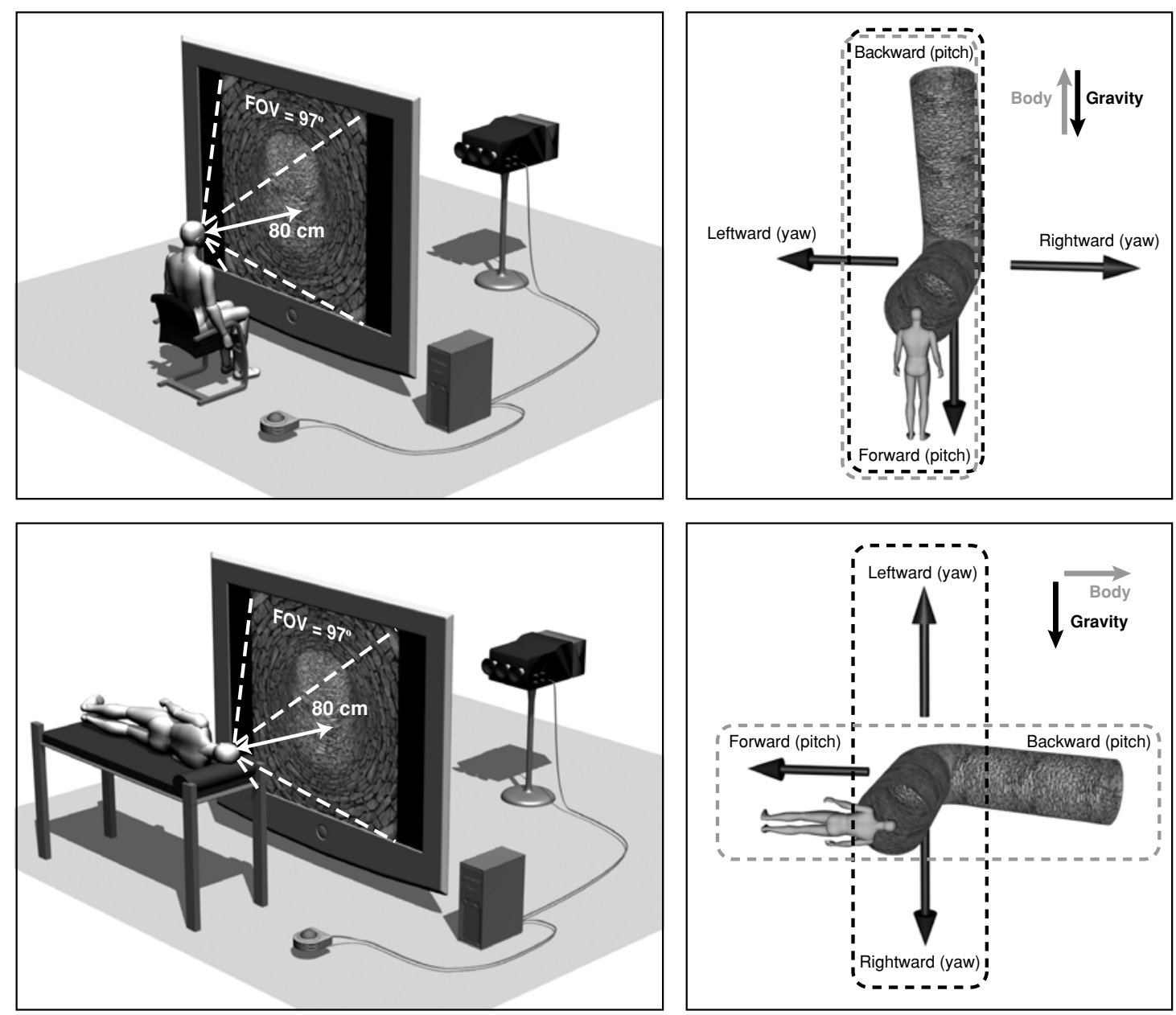

Figure 1. The left panels show the experimental setup for both the upright (top) and the lying on the side (bottom) conditions. The subjects' line of sight was centered on a translucent screen subtending $97^{\circ}$ of horizontal and vertical field of view (FOV). They used a trackball for the reproduction task. A PC computer with a GeForce2 video card generated the virtual displacements projected onto the screen. The right panels give the turn direction conventions and how asymmetrical factors are dissociated in two groups according to whether they are defined in a body- or an earth-centric reference frame.

for all the subjects. If we suppose that an observer measuring $1.75 \mathrm{~m}$ in height is walking along the floor of the corridor, the simulated motion speed would be $2.21 \mathrm{~m} / \mathrm{sec}$ (around $8 \mathrm{~km} / \mathrm{h}$ ), which corresponds to a fast walking speed for humans.

Data analysis. Errors for reproduced angles and response latencies on each trial were recorded. The response latencies measured in the reproduction task corresponded to the time that elapsed between the initial presentation of the corridor with $0^{\circ}$ of deviation and the moment that the subjects pressed the button to validate the reproduced turn. We analyzed the relative angular error (percentage of the actual turn amplitude), rather than the absolute angular error, because we considered that a $20^{\circ}$ error on a $45^{\circ}$ turn was not equivalent to a $20^{\circ}$ error on a $157.5^{\circ}$ turn. In the first case, it would correspond to a relative error of $44.4 \%$, and in the second case, to a relative error of $12.7 \%$. Angular error was signed according to the following convention: positive if the reproduced angle was overestimated (overshot) and negative if it was underestimated (undershot).

A 2 (body position) $\times 2$ (turn orientation) $\times 2$ (turn direction) $\times$ 6 (turn angle) repeated measures ANOVA design was used, with body position (upright or lying on the side), turn orientation (yaw or pitch), turn direction (leftward or rightward for yaw and forward or backward for pitch), and turn angle $\left(45^{\circ}, 67.5^{\circ}, 90^{\circ}, 112.5^{\circ}, 135^{\circ}\right.$, or $157.5^{\circ}$ ) as the within-subjects factors. The dependent variables were relative angular error, differential errors, and response latency. In addition, we compared the level of asymmetry observed for the reproduction performance of pitch and yaw across conditions. These were defined as the forward minus the backward and the rightward minus the leftward relative errors, respectively. Post hoc analyses were performed with Tukey's test.

\section{Results}

Reproduction accuracy. When the subjects were seated upright, the error curves of the reproduced angles for yaw turns were not statistically different (see Figure 3A) [turn direction $\times$ turn angle interaction, $F(5,75)=1.35$, $p=.25]$. As one could expect for the reproduction of a perceived psychophysical variable without feedback, we observed a range effect that tended to push estimation of both small and large angles closer to the same average value. The error curves were not symmetrical for back- 
A) Inside View During Exploration

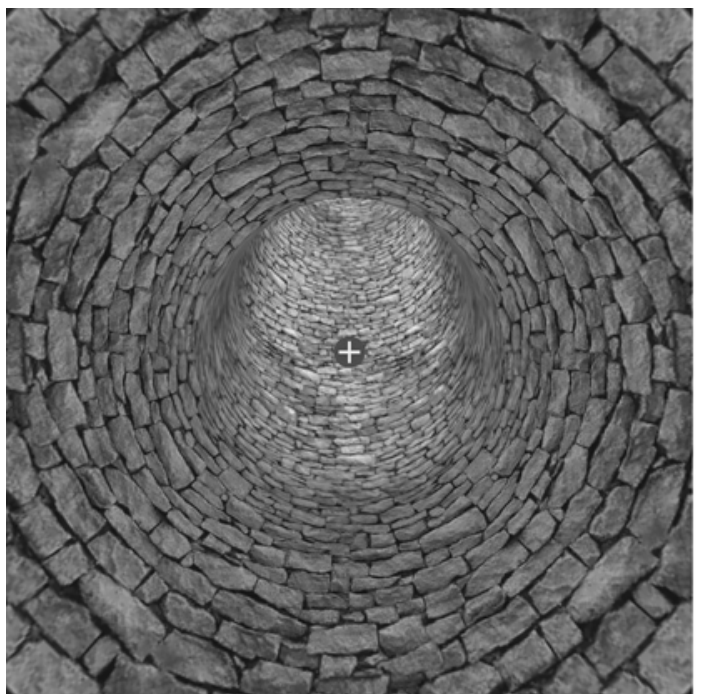

B) Reproduction Task

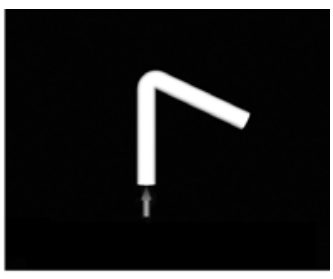

Yaw turns: top view

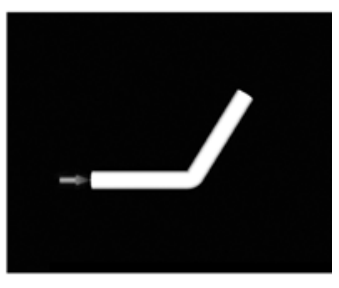

Pitch turns: side view

Figure 2. (A) An inside static view of a corridor explored by the subjects (resolution of $1,200 \times 1,200$ pixels at a frame rate of $60 \mathrm{~Hz}$ ). The perspective correction was adjusted to the real field of view experienced. The central fixation cross used in Experiment 2 is drawn according to its relative size in the screen. (B) Two outside views of the corridor as seen during the reproduction task in the upright position for yaw turns (top) and pitch turns (bottom). The arrow indicates the point of entry.

ward and forward pitch turns (see Figure 3B), and this interaction was statistically significant [turn direction $X$ turn angle interaction, $F(5,75)=12.83, p<.001]$. Error curves for forward turns decreased continuously with increasing turn angle, whereas they remained stable for backward turns. Errors over all angles were 16.9\% for forward turns and $-1.8 \%$ for backward turns, with a significant difference of $18.7 \%$ [turn direction main effect, $F(1,15)=24.93, p<.001]$. Post hoc tests revealed that this interaction came mainly from the differences in the processing of $45^{\circ}$ turns $(p<.001)$ and $67.5^{\circ}$ turns $(p<.001)$.

When the subjects were lying on their right sides in a $90^{\circ}$ roll position, error curves for yaw turns diverged (see Figure 3C). Rightward turns - that is, corridors that curved downward with respect to gravity-were overestimated (mean error $=12.1 \%$ ) to a greater degree than were leftward turns (mean error $=1.1 \%$ ), by about $11.0 \%$ on average [turn direction main effect, $F(1,15)=21.95$, $p<.001]$. Post hoc tests confirmed that the errors for leftward and rightward turns differed statistically for turn angles of $45^{\circ}(p<.001), 67.5^{\circ}(p<.001)$, and $90^{\circ}(p<$ $.02)$. Error curves recorded for pitch turns when the subjects were lying on their sides were still not symmetrical (see Figure 3D) and presented a significant difference between pitch forward and pitch backward curves [turn direction $\times$ turn angle interaction, $F(5,75)=15.05, p<$ $.001]$. The difference between the overall relative errors for forward and backward turns was, in this case, $19.5 \%$ [turn direction main effect, $F(1,15)=38.74, p<.001$ ] Post hoc tests showed that $45^{\circ}$ turns $(p<.001), 67.5^{\circ}$ turns $(p<.001)$, and $90^{\circ}$ turns $(p<.005)$ contributed to this interaction.

Reproduction latency. Response latencies were affected by body position [body position main effect, $F(1,15)=13.75, p<.003]$. They were significantly higher when the subjects were lying on their sides than when they were upright for both yaw turns $(4,400$ vs. 3,900 msec, $p<.05)$ and pitch turns (5,250 vs. $4,100 \mathrm{msec}, p<.001)$. The increased latency when the subjects were $90^{\circ}$ rolltilted can be explained by the fact that handling the trackball in this position can be less natural than it is when one is upright. There was no other significant effect of the experimental factors on latencies.

\section{Discussion}

In the upright position, the subjects produced errors in the reproduction of yaw turns that were identical for leftward and rightward turns. In other words, the perceived angle for a given leftward turn and its rightward turn counterpart were the same (see Figure 3A). These results were expected, given that visual and vestibular receptors are symmetrical with regard to the midsagittal plane. In this body position, gravity will act symmetrically, if at all, whether one is turning left or right, and furthermore, it is aligned with the body longitudinal axis, which is the natural orientation with respect to the body during human locomotion. In contrast, error curves for pitch turns when the subjects were upright were not symmetrical (see Figure 3B), meaning that for the same angle, forward and backward turns were not perceived as being equivalent. Again, this finding fits with the predictions outlined in the introduc- 
UPRIGHT POSITION

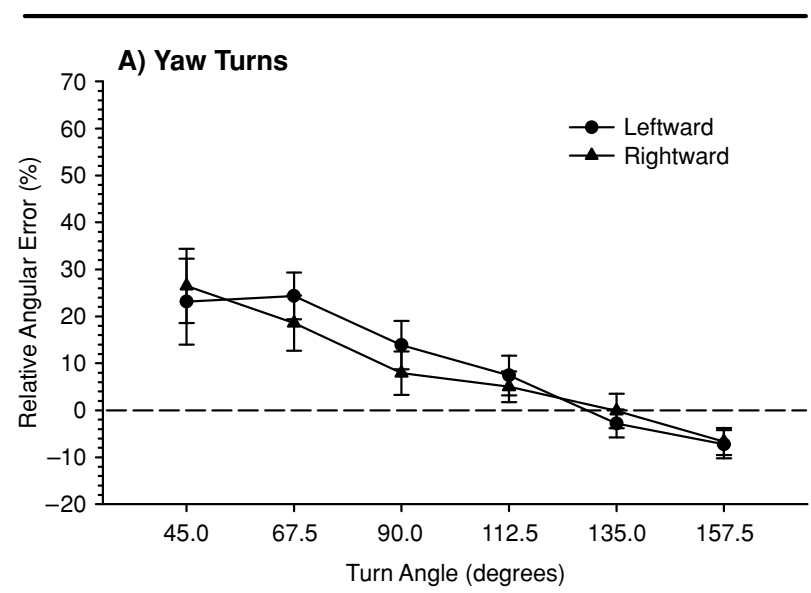

B) Pitch Turns

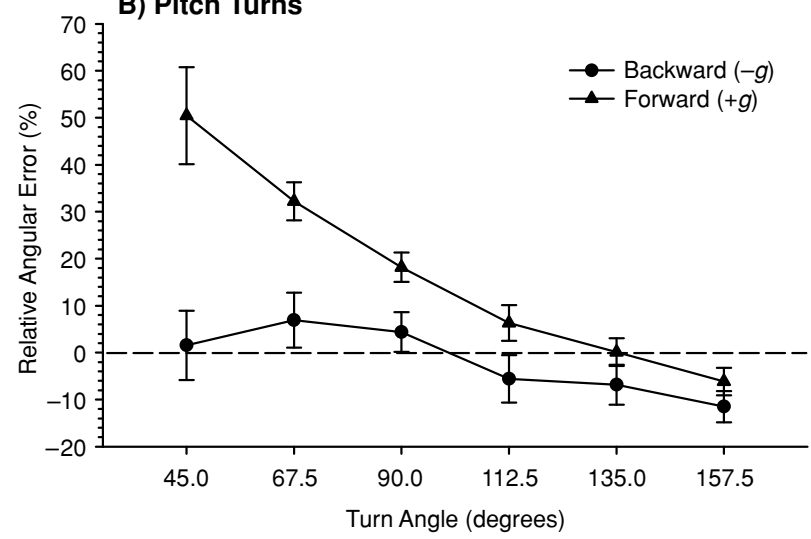

RECLINED POSITION
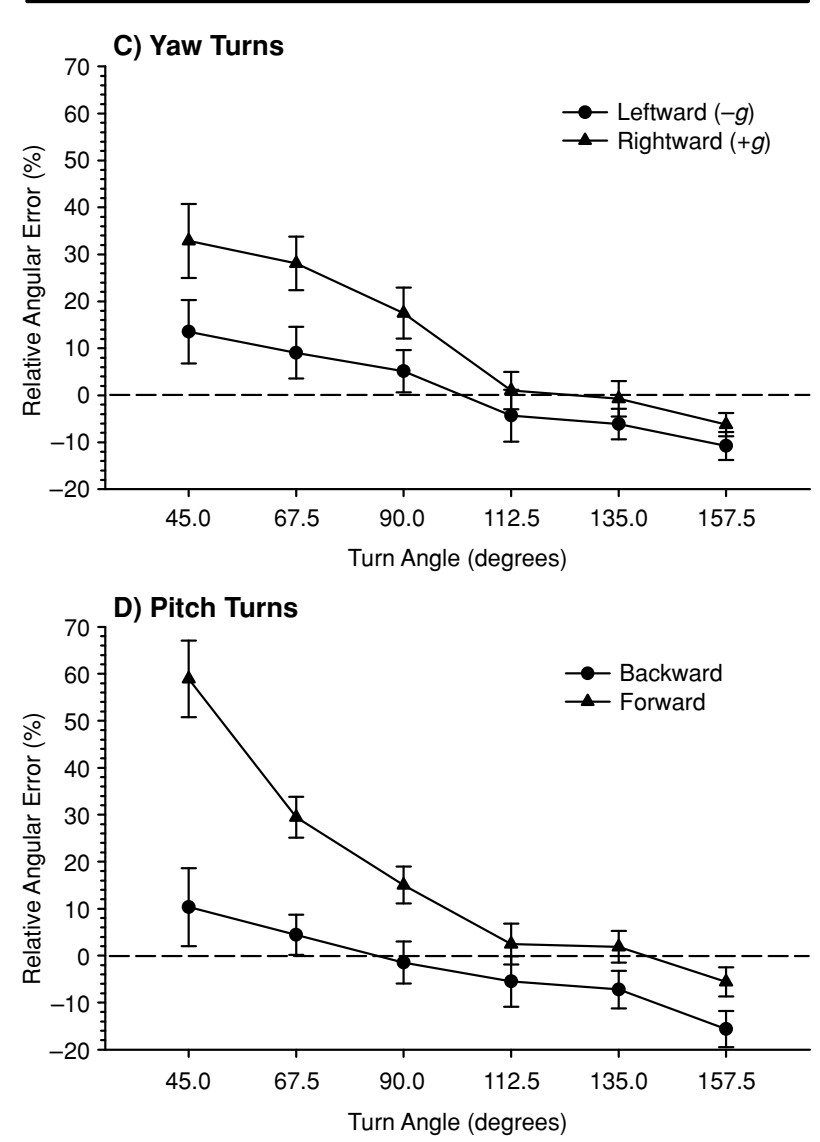

Figure 3. The relative angular errors of perceived continuous yaw and pitch turns when the subjects were seated upright $(A$ and $B)$ and when they lay on their right sides (C and D). Dashed line: overshot/undershot frontier. Error bars indicate standard errors $(N=16)$.

tion and is consistent with the results of previous studies on the perception of self-orientation (Cohen \& Larson, 1974) and vection-induced pitch (Young et al., 1975).

By having the subjects lie on their sides in a $90^{\circ}$ roll position, we modified the influence of gravity for both yaw and pitch turns. Yaw turns became susceptible to a direct effect of gravity on perception, whereas it was suppressed for the perception of pitch turns. In this way, we dissociated the sources of influence related to the visual reference frame from those related to the inertial reference frame.

When the subjects lay on their sides, errors in the reproduction of yaw turns were no longer symmetrical for leftward and rightward turns. In this position, leftward turns of less than $90^{\circ}$ were overestimated to a lesser degree than were the equivalent rightward turns (see Figure 3C). Therefore, polarizing the turning direction with respect to gravity has a clear influence on the perception of yaw turns. In contrast, suppressing the sensed gravity that, in natural upright postures, polarizes pitch turns did not affect the performance obtained for either forward or backward turns; that is, forward and backward perception of pitch turns showed the same level of asymmetry in the reclining position (see Figure 3D). This suggests that the influences defined in the visual egocentric reference frame were sufficiently strong to induce the observed asymmetrical responses in pitch perception when the subjects were upright, and removing inertial cues did not reduce the observed pitch asymmetry.

Note that the asymmetry introduced in yaw turn perception had a nature different from the one observed for pitch turns [significant interaction with the turn angle, $F(3,75)=3.74, p<.005]$. These results are consistent with the hypothesis of distinct perceptual cues, both from the visual egocentric reference frame and from the inertial earth-centric reference frame, either of which is sufficient to induce asymmetry in the perception of "upward" versus "downward" turns. Since both motion cues (optic flow) and static cues (corridor's geometry) were available in Experiment 1, the five potential asymmetry factors that were identified in the introduction could play a role in the resulting perception of turns (Factors $1-5$ in Table 1).

Path integration mechanisms (Factors 1 and 3). The temporal processing of motion cues, corresponding to the multisensory integration of optic flow and of vestibular and somatosensory information, are involved in spatial orientation mechanisms commonly referred to as 
path integration mechanisms. The major contribution of these in the estimation of one's orientation and position during self-motion is well established. The visual cues and inertial cues included in this integration process have specific asymmetrical influences according to the orientation of gravity and human physiological and functional characteristics. For instance, eye movements, whether spontaneous or not, generated by the optic flow resulting from the exploration of corridors might interfere with the path integration mechanisms. Indeed, summing the successive displacements performed during the slow phases of optokinetic nystagmus (OKN; spontaneous movements) or summing the successive jumps to particular targets in the scene (directed target pursuit) could provide a good indication of the amplitude of the actual turn. Many previous studies have reported significant asymmetries in mammalian eye movements during vertical stimulation. Matsuo, Cohen, Raphan, de Jong, and Henn (1979) reported asymmetries in the vertical OKN of monkeys, which would result from a stimulus velocity storage deficit. Tilting the animal sideways resulted in a tilted asymmetry, showing that this effect is defined in the egocentric reference frame. Similar results were also found in humans for the nystagmus (Clément, Vieville, Lestienne, \& Berthoz, 1986), the vestibulo-ocular reflex (Grigorova \& Kornilova, 1996), and eye pursuit movements (André-Deshays et al., 1993). Since the velocity of the visual surround is improperly stored for upward OKN/pursuit, which in our experiment corresponds to forward pitch rotations, this process is no longer reliable and could be responsible for the observed errors for forward turn angles. In contrast, this velocity storage problem is not encountered for downward OKN/ pursuit, thus providing an effective process for estimating backward turns. The physiological asymmetries of vertical eye movements probably contributed to the asymmetry observed in the perception of pitch turns whether the subject was upright or lying down.

Gravity could also take part in the cues that are integrated during self-motion in order to infer body orientation changes. The otolithic system and the somatosensory receptors are symmetrically positioned across the median plane, but not across any sagittal plane (Wilson \& Melvill Jones, 1979). Thus, gravity could not produce a different effect on the perception of leftward and rightward turns. In contrast, because of the polarized activation of these sensors along with the earth-vertical axis, such an input in the path integration mechanisms could be responsible for the asymmetry in the perception of pitch turns in the upright position and in the perception of yaw turns in the reclined position. However, the level of asymmetry between the perception of forward and backward turns was not statistically different between the upright and the reclined positions. We concluded that graviceptor information telling the subject that pitch turns were now in the horizontal plane was not strong enough to overcome other cues in the perception of pitch turns. The latter were sufficient to create the asymmetry, even though direct gravity cues were no longer involved. Moreover, in our experiments, the motion simulation was purely visual, and gravity orientation changes were not consistent with the visual orientation changes. Therefore, because gravity information was not relevant for motion perception, it might not have contributed to the path integration process.

Processing of geometrical cues (Factor 4). Because of the functional adaptation of humans to terrestrial gravity, estimating the amplitude of turns from the geometrical shape of the environment can be different for turns going up and down. Processing these cues involves visual scanning of the environment, and therefore, the perceptual asymmetry that can be introduced is defined relative to the observer's orientation. In other words, this influence in our experiment could have contributed to the observed difference in the perception of forward and downward turns when the subjects were upright or reclined.

Scene navigability (Factors 2 and 5). At a higher cognitive-processing level, sensing gravity can be subjected to a top-down influence on turn perception. Indeed, the potential consequences afforded by the visual scene may influence perception. For instance, after the experiments, the subjects often reported that they had felt as if they were going to fall when they were turning downward (in the direction of gravity). The fact of knowing (i.e., by sensing the orientation of gravity) that one can fall when going down could affect the angle estimations by exaggerating the perceived degree of turn. In principle, this fearof-falling factor is related to inertial cues (Type 2; Table 1) and, therefore, could have contributed to the asymmetry of pitch turns observed when the subjects were upright and to the asymmetry introduced in the perception of yaw turns observed when they reclined. But this assessment of scene affordance could also be implemented with respect to the visual scene- that is, in an egocentric reference frame (Type 5) - which would result in effects on pitch turns in both the upright and the reclining positions.

We have identified a number of factors that could have been the source of the asymmetry observed in the perception of pitch forward and pitch backward turns. Experiments 2 and 3 were designed to assess the role of each possible factor. Asymmetrical eye movements (notably, OKN) could play a role, but only in the presence of a moving scene and when the eyes can move freely. These two conditions were selectively suppressed in Experiments 2 (no eye movements) and 3 (no visual motion). Effective perception of geometrical cues requires scanning of the visual scene. The assessment of the potential role of this factor was tested by suppressing eye movements in Experiment 2, but not in Experiment 3. Finally, the top-down influence of scene affordance acts a priori, independently of static or motion cues, and we would therefore expect to find it in both of the following experiments.

\section{EXPERIMENT 2 Motion Cues for Turn Perception}

In Experiment 1, asymmetry was found in the perception of pitch turns performed in a virtual visual environment. By laying subjects down on their sides, we showed that the direction of gravity has a direct influence on the 
perception of turns. Nevertheless, the factors acting in an earth-centric reference frame (gravity input, both in the path integration mechanisms and in the estimation of the scene navigability) are not solely responsible for the observed asymmetry in pitch turns. Indeed, modifying the subjects' body position and, thus, the direction of inertial cues did not change the level of asymmetry between forward and backward pitch estimations. We have already stated that there is a strong asymmetry in most vertical eye movements and, particularly, in velocity storage for upward and downward OKN. Since one possible way to extract the amount of turning performed dynamically could be the summation of slow-phase deviations of the eye during the OKN induced by the optic flow, we hypothesized that most of the pitch asymmetry observed in Experiment 1 could have been due to the physiological asymmetry of upward and downward eye movements performed during the visual stimulation. In a second experiment, subjects were asked to keep their gaze on a fixation point during the whole of the simulated visual motion. This manipulation suppressed both the spontaneous OKN induced by the optic flow and visual saccades that could otherwise have been performed in order to visually scan the shape of the bending corridor. Indeed, because the subjects had to fixate a central point, the geometrical cues that were in the peripheral vision could not be correctly perceived, although motion cues could still be processed (Cutting, Vishton, Flückiger, Baumberger, \& Gerndt, 1997). Thus, in this experiment, the processing of visual motion cues could still influence perception, but not that of static geometrical cues. We therefore examined whether eye movements, spontaneous or not, were responsible for the observed differences between the perception of forward and backward turns.

\section{Method}

Sixteen new naive subjects ( 10 men and 6 women), between 22 and 30 years of age, participated in the experiment. All except 2 were right-handed. They all gave prior written consent before starting this investigation.

In Experiment 2, we used the same apparatus as that in Experiment 1 , but this time a monocular eyetracker (VEONYS) recorded movements of the left eye during visual stimulation. During the whole displacement simulation along the corridor, a fixation point was presented in the middle of the screen. The fixation point consisted of a white cross in the middle of a red disk covering $5.9^{\circ}$ of solid angle (see Figure 1). The procedure was also the same as that in Experiment 1, except that the angles of the turns studied in this experiment ranged from $45^{\circ}$ to $112.5^{\circ}$ in increments of $22.5^{\circ}$, resulting in 16-trial blocks. We limited the angles studied to $112.5^{\circ}$ because the results of the previous experiment revealed that there was little difference between forward and backward turn perception for very large angles. The subjects were asked to keep looking at the fixation point during the whole of the visual exploration and to avoid blinking before and during the turns. The same reproduction task followed each exploration (see Figure 2B). The full experiment lasted approximately $75 \mathrm{~min}$ and was done in a single session.

\section{Results}

Eye movements. For each trial, movements of the left eye were recorded at a sampling rate of $50 \mathrm{~Hz}$. Only $2.0 \% \pm 2.6 \%(M \pm S D)$ of the trials were performed with eye movements before or during the visual scene rotation: $1.3 \%$ resulting from spontaneous movements (OKN and single saccades of more than $4^{\circ}$ of amplitude) and $0.7 \%$ resulting from controlled movements (possibly, the tracking of geometrical cues). Given the low rate of trials on which the subjects were unable to refrain from making eye movements, we considered that these were unlikely to have caused much interference with the average performance, and therefore, we left them in the statistical analysis.

Reproduction accuracy. When the subjects were seated upright, the relative error curves of leftward and rightward yaw turns observed with a fixation point were not different (see Figure 4A). As in Experiment 1, the range effect was moderate, and the perceived yaw turn angles were fairly close to the simulated turn angle. In contrast to the previous results, the relative error curves for pitch turns were nearly identical for forward and backward turns (see Figure 4B). Indeed, neither a turn direction effect nor a turn direction $X$ turn angle interaction was observed.

When the subjects were lying on their sides in a $90^{\circ}$ roll position, error curves for rightward and leftward turns were separated, even though the eyes were kept static (see Figure 4C). Again, rightward turns (mean error $=-3.7 \%$ ) were overestimated more than were leftward turns (mean error $=-15.9 \%$ ) by about $12.2 \%$, on average [turn direction main effect, $F(1,15)=12.99, p<.003]$. Post hoc tests revealed that only the leftward and rightward $45^{\circ}$ turns were statistically different $(p<.001)$. The relative errors recorded with a fixation point when the subjects were lying on their sides were no longer symmetrical (see Figure 4D). Forward and backward mean error difference was approximately $11.5 \%$ [turn direction main effect, $F(1,15)=9.36, p<.01]$. Post hoc tests revealed significant differences in the average errors observed between forward and backward $45^{\circ}$ turns $(p<.01)$ and $67.5^{\circ}$ turns $(p<.006)$.

\section{Discussion}

For the upright position, the response errors for the perception of yaw turns when the eyes were kept static were again symmetrical with regard to the turning direction. Turns were underestimated by $31.1 \%$, in comparison with the overestimations of yaw turns observed in Experiment 1 for the same deviation angles (from $45^{\circ}$ to $112.5^{\circ}$ only). Allowing observers to use geometrical cues and to move their eyes freely, as in Experiment 1, seems to increase the perceived amplitude of yaw turns. The errors measured for pitch turns were, in this case, quite symmetrical (no statistical differences), and small turns were overestimated to a greater degree than were the equivalent yaw turns $\left(13.9 \%\right.$ more for $45^{\circ}$ turns, $\left.p<.003\right)$. The main differences from Experiment 1 were the suppression of both eye movements (factor of Type 3 ) and geometrical information (Factor 4) as cues available to estimate the turn angle. Since, in this experiment, there was no difference between the perception of forward and backward turns, we conclude that the main factor responsible for the perceptual asymmetry observed in Experiment 1 was ei- 
UPRIGHT POSITION
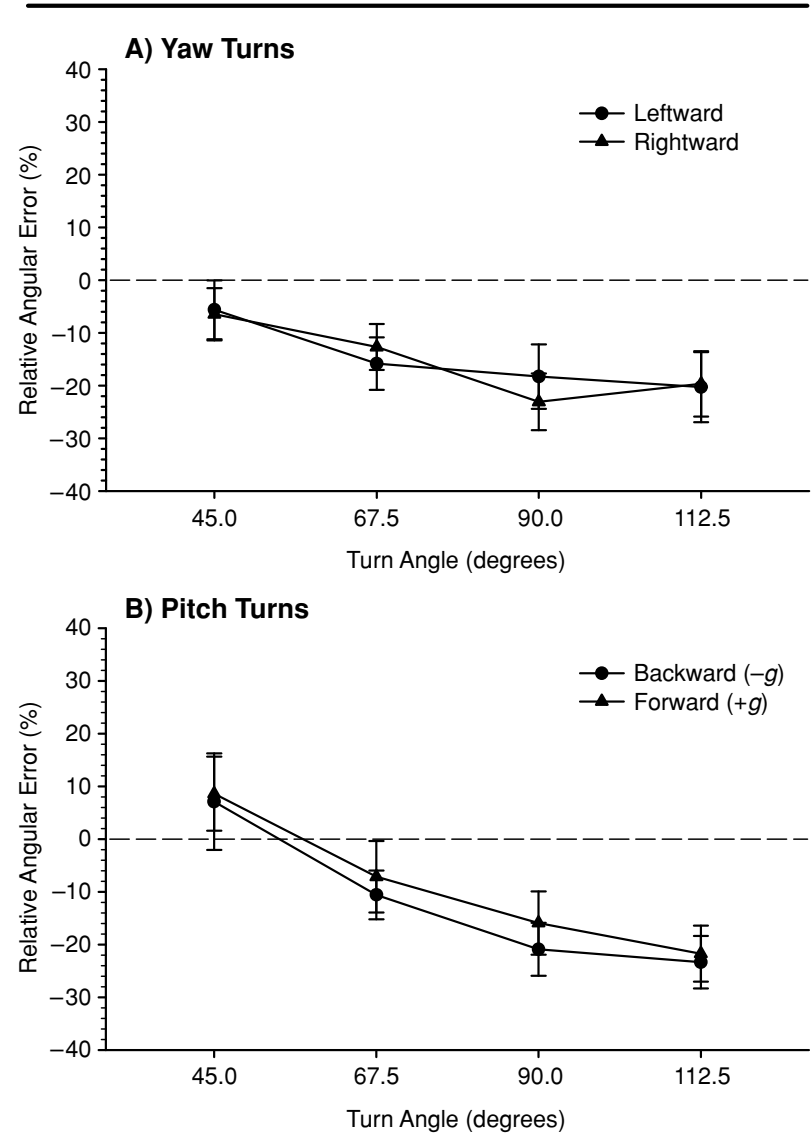

RECLINED POSITION
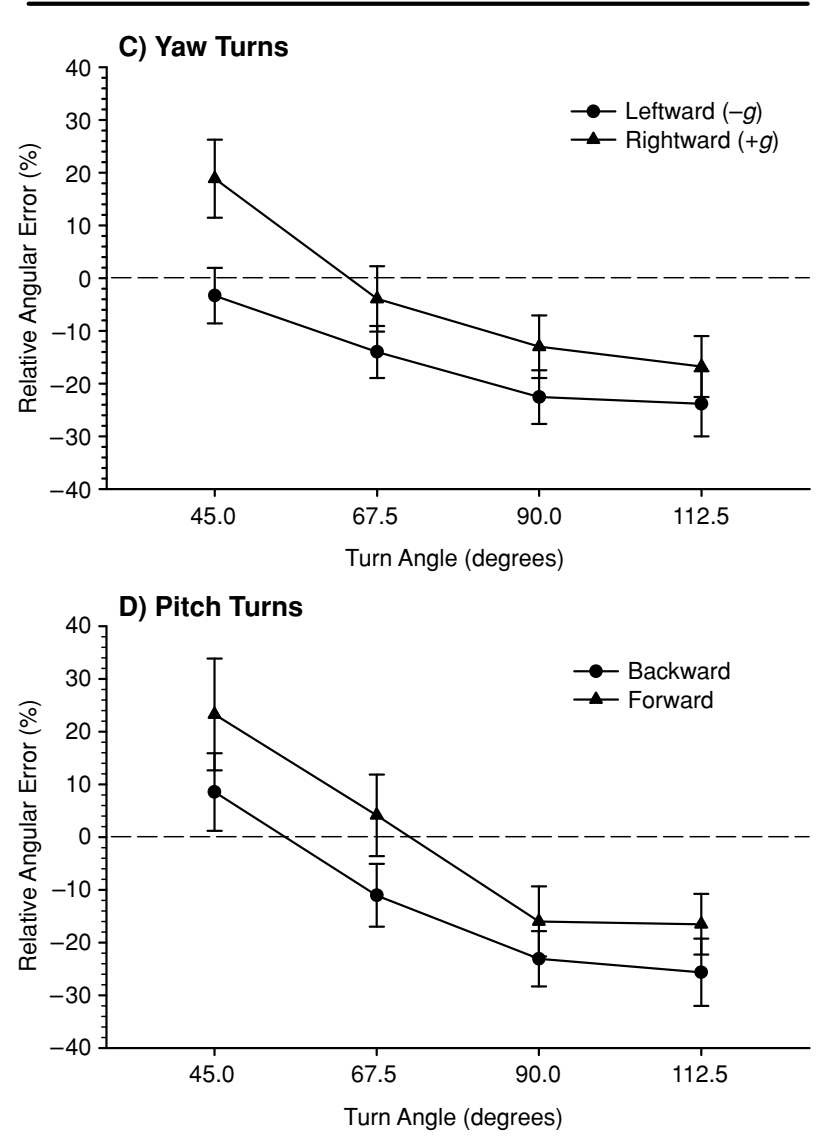

Figure 4. The relative angular errors of perceived yaw and pitch turns with a fixation point when the subjects were seated upright (A and B) and when they lay on their right sides (C and D). Dashed line: overshot/undershot frontier. Error bars indicate standard errors $(N=16)$.

ther the physiological asymmetry of upward versus downward eye movements - namely, OKN_or the processing of 3-D geometrical cues. Experiment 3 made it possible to disambiguate this last point.

When the subjects were lying on their sides, the modified orientation of gravity still had a significant influence on the perception of yaw-curving corridors. Rightward turns (in the direction of gravity) were globally overestimated by $12.2 \%$, in comparison with the same leftward turns, which is similar to the difference of $14.0 \%$ observed in Experiment 1 for the same deviation angles (from $45^{\circ}$ to $112.5^{\circ}$ only). This is consistent with the predictions noted in Table 1. Indeed, in these two experiments, the same asymmetry factors could act during the perception of yaw turns in the reclined position (Factors 1 and 2). Interestingly, for the reclining position, the errors measured in the perception of pitch turns also became asymmetrical: Forward turns were underestimated less, by $11.5 \%$, than were backward turns. These results indicate that the reclining subjects altered the sensed gravity orientation and substantially modified the perception of turns: Pitch turns were affected by the suppression of the inertial related fac- tors, whereas yaw turns were affected by their addition. On the other hand, for the reclined position, only the processing of visual-related cues can introduce an asymmetry in the perception of pitch turns - namely, the remaining asymmetrical processing of optic flow in the path integration mechanisms (Factor 3, excluding eye movements) and the evaluation of visual scene navigability (Factor 5).

The level of asymmetry in the reclined position was equivalent for the perception of pitch and yaw turns (the turn direction main effect and the turn direction $\times$ turn angle interaction were not significant). Therefore, visionrelated and inertia-related asymmetry factor groups had approximately the same strength. For the reclined position, we found that the inertia-related factors tended to increase the perception of rightward turns (toward the ground), as compared with leftward turns, and the vision-related factors tended to increase the perception of forward turns (toward the feet), as compared with backward turns. Each of these factor groups introduced an asymmetry that should go in the same direction in the upright position. Indeed, in the upright body position, these groups should tend to increase the forward turns (toward both gravity and the 
feet), as compared with backward turns. Curiously, there was no significant difference between forward and backward pitch turns in the upright position.

The sizes of these effects $(12.2 \%$ and $11.5 \%)$ are not comparable to the level of asymmetry observed for pitch turns when all the cues were available $(24.5 \%)$. This apparent nonadditivity might have been partially due to the sensitivity of these weaker effects to small context variations, which we can try to explain with the following. In the upright position, the otolithic stimulation was not consistent with the simulated visual motion of pitch turns. This was no longer the case for pitch turns in the reclined position, where rotations were performed around the gravity axis and no otolithic signal was expected. This noticeable perceptual conflict in the upright position could have led to an inhibition of the otolithic signal in the path integration mechanisms, which would then have modified its influence on turn perception (Factors 1 and 4). On the other hand, changing substantially the somatosensory postural feedbacks from an upright position to a reclined position might also affect the evaluation of scene navigability. Indeed, one might be more likely to evaluate a potential risk of falling during rotations toward the ground when lying on the side than when sitting on a chair (Factor 2).

These findings provide strong evidence in support of the idea that there are two groups of effects, defined either in an earth-centric reference frame or in an egocentric reference frame, that influence the perception of turns. The first group relates to the inertial cues and affects yaw, rather than pitch, turns once subjects are in the reclined position, whereas the second group relates to the visual cues and always affects pitch turns independently of the direction of gravity. Tilting subjects on their sides, although nonrealistic with regard to the vestibular stimulation, allowed us to highlight some hidden influences that might otherwise be inhibited.

\section{EXPERIMENT 3 Static Cues for Turn Perception}

A third experiment was designed to deprive subjects of the possibility of using motion cues to estimate the angle of turn. This time, only static pictures of turns were presented, providing the subjects with only static geometrical information on the bending corridors. Since there was no turning motion in Experiment 3, all influences on the processing of motion cues, such as the path integration in the previous experiments, were now absent. On the one hand, this new experiment allowed us to check whether earth-centric cues, such as sensing gravity, would affect the static perception of turns. Indeed, cognitive cues, such as the evaluation of scene navigability and the processing of geometrical cues, should act independently of the optic flow generated by motion. On the other hand, it allowed us to determine the relative contribution of static information versus continuous motion information in the perceptual asymmetry observed in Experiment 1 for pitch turns. This experiment made it possible to determine whether the asymmetrical physiology of the OKN or the use of geometrical cues was the main factor affecting turn perception.

\section{Method}

Sixteen new naive subjects ( 10 men and 6 women), between 22 and 37 years of age, participated in the experiment. All except 1 were right-handed. They all gave prior written consent before starting this investigation.

The experiment used exactly the same apparatus as that in Experiment 1 , except that this time, static views of the corridors were shown, instead of a continuous motion simulation along the corridor. The static views of the turns were taken at the position that just preceded the onset of virtual gaze rotation in the previous experiments (as in Figure 2A). The procedure was the same as that in Experiment 2, with angles of turns ranging from $45^{\circ}$ to $112.5^{\circ}$ in increments of $22.5^{\circ}$ and blocks of 16 trials. In Experiment 3, we studied angles only up to $112.5^{\circ}$, because in the static pictures, there was no visible difference for turn angles greater than $112.5^{\circ}$. The static views of the turns were displayed for $2.7 \mathrm{sec}$, followed by the same reproduction task as that in the previous experiments (see Figure 2B). The presentation duration corresponded to the length of time that the subjects could see the corridor before starting to turn in Experiment 1. The full experiment lasted approximately $50 \mathrm{~min}$ and was done in a single session.

\section{Results}

Reproduction accuracy. As in the previous experiments, when the subjects were seated upright, the error curves for responses to static yaw turns were symmetrical: No statistical difference between leftward and rightward turns was found (see Figure 5A). As in Experiment 1, when the subjects were seated upright, the curves of relative angular errors were not symmetrical for static forward and backward turns (see Figure 5B). The mean difference between forward and backward turn relative errors was $8.3 \%$ [turn direction main effect, $F(1,15)=10.30, p<$ $.006]$, and the turn direction $\times$ turn angle interaction was statistically significant $[F(3,45)=5.10, p<.004]$. Post hoc tests showed that this interaction was due to the difference in the average error observed for forward and backward $45^{\circ}$ turns $(p<.001)$.

When the subjects were lying on their sides in a $90^{\circ}$ roll position, the error curves for rightward and leftward turns were again separated (see Figure 5C). Leftward turns $(-14.2 \%$ of error) were underestimated more than were rightward turns $(-0.1 \%$ of error) by around $14.1 \%$, on average [turn direction main effect, $F(1,15)=32.79, p<$ $.001]$. The turn direction $\times$ turn angle interaction was not significant, indicating that the error curves were nearly parallel across stimulus angles. Post hoc tests confirmed that the error for leftward and rightward turns differed statistically for every turn angle $\left(p<.001\right.$ for $45^{\circ}$ and $67.5^{\circ}, p<.003$ for $90^{\circ}$, and $p<.04$ for $112.5^{\circ}$ ). The relative error recorded for static pitch turns when the subjects were lying on their sides was still not symmetrical (see Figure 5D). Forward and backward mean error difference was, this time, approximately $13.2 \%$ [turn direction main effect, $F(1,15)=15.43, p<.002]$. The effect of the turn angle varied significantly with the turn direction [turn direction $\times$ turn angle interaction, $F(3,45)=3.98, p<$ $.014]$. The post hoc test revealed that this interaction came 
UPRIGHT POSITION
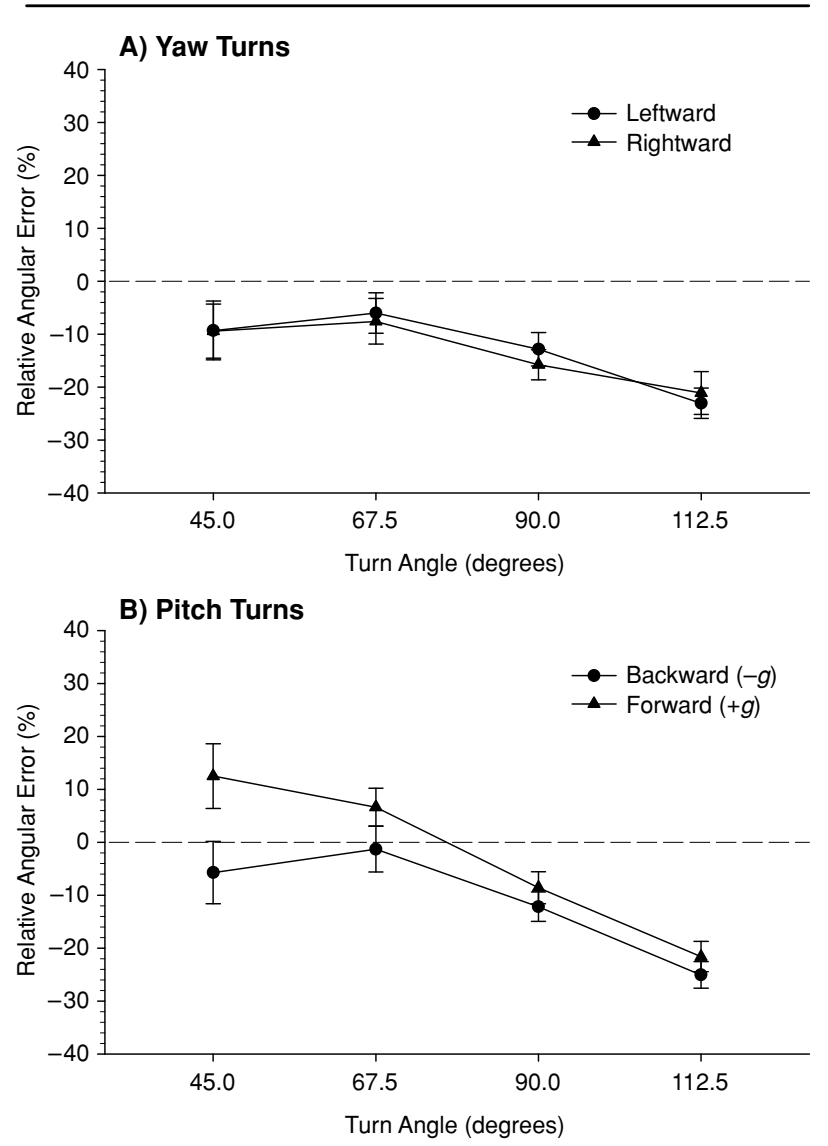

RECLINED POSITION
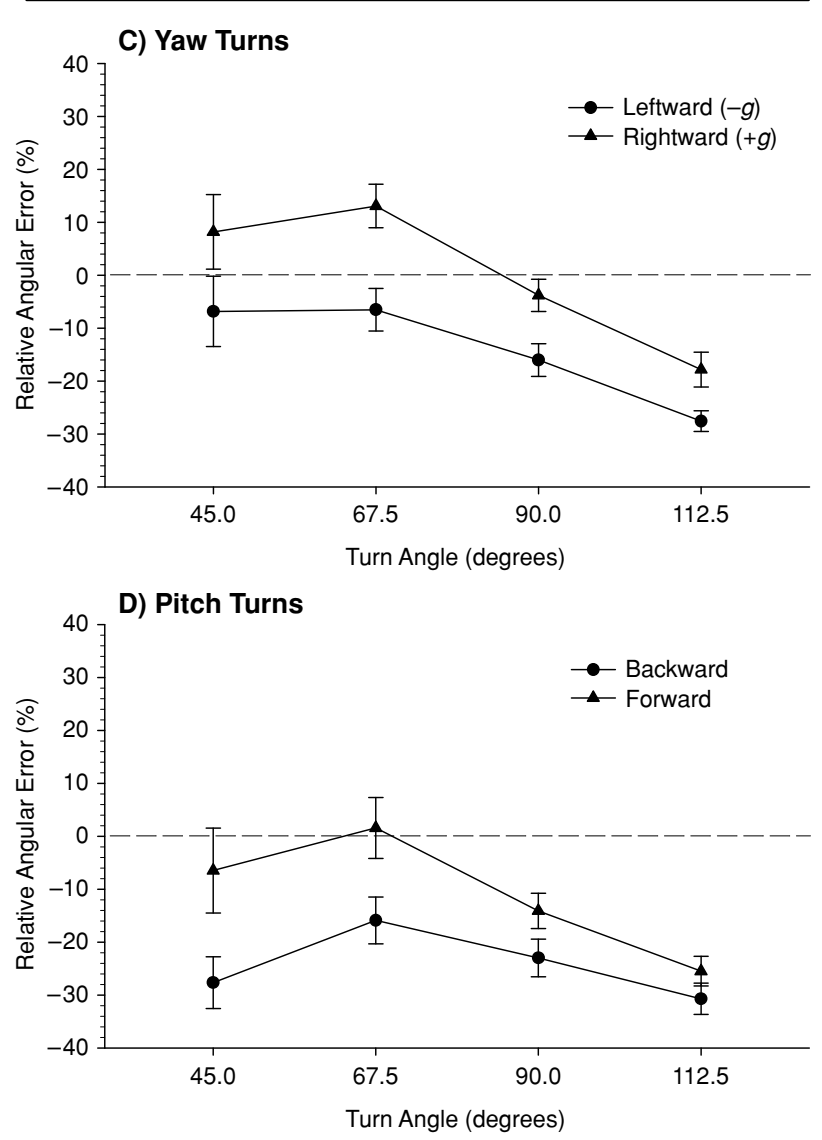

Figure 5. The relative angular errors of perceived static yaw and pitch turns when the subjects were seated upright (A and B) and when they lay on their right sides (C and D). Dashed line: overshot/undershot frontier. Error bars indicate standard errors $(N=16)$.

mainly from the differences in the average errors observed between forward and backward $45^{\circ}$ turns and $67.5^{\circ}$ turns $(p<.001)$.

\section{Discussion}

For the upright position, the errors measured for the perception of static pictures of yaw turns were, as in the previous experiments, symmetrical between leftward and rightward turns. When comparisons were made for the same deviation angles in Experiment 1, turn perception was underestimated by about $29 \%$. This shows that when motion cues were available throughout the corridor, the estimation of turn increased. Therefore, the corridor's deviation angles were perceived as being larger when the combination of motion and static cues was available than when each one was taken separately.

The perception of forward and backward turns differed significantly only for the $45^{\circ}$ turns; thus, only residual asymmetry persisted, in comparison with that observed in Experiment 1. We showed previously that either the physiological asymmetry of vertical eye movements or the processing of geometrical cues was responsible for the pitch perceptual asymmetry observed in Experiment 1.
In Experiment 3, in which the optic flow was absent, we found that once the subsequent OKN was suppressed, perception with the remaining geometrical cues led to a substantially smaller residual asymmetry. Therefore, the possibility of using geometrical cues could not have contributed significantly to the asymmetry of pitch turns observed in Experiment 1, and it was, rather, the upward versus downward OKN physiological difference that introduced this asymmetry.

When the subjects lay on their sides, the orientation of gravity still influenced their perception of static views of yaw-curving corridors. Since, in Experiment 3, there were no motion cues to process, only the processing of the inertia-related cognitive cues could have been responsible for the measured asymmetry. The offset difference between leftward and rightward error curves was about $14.1 \%$, on average, which is similar to that found in both Experiments 1 and 2 for the same angles $(14.0 \%$ and $12.2 \%$, respectively). Therefore, the evaluation of scene navigability according to terrestrial gravity (Factor 2 in Table 1) was probably the main factor responsible for the same asymmetry observed in Experiments 1 and 2. This is another indication supporting the finding that the 
inertia-related motion cues (Factor 1) had no asymmetrical influence in the perception of turns defined in the earth-vertical plane. Indeed, in our experiments, the latter was not consistent with the actual simulated motion. The perception of static views of pitch turns in the reclining position led to asymmetrical errors with regard to the turn direction, forward turns being overestimated, in comparison with the same backward turns. This asymmetry was stronger than the one observed when the subjects were seated upright [body position main effect, $F(1,15)=5.14$, $p<.04$, independent of the turn angle]. As for Experiment 2 , the inertia-related and vision-related groups of asymmetry factors were again acting on turn perception. Nevertheless, as the strength of their effect was reduced, they apparently became sensitive to the distinct somatosensory contexts between the upright and the reclined positions. In fact, in this experiment, there was no longer a conflict between the vestibular activation and the actual simulated position in the tunnels. This could have resulted in the nonlinear additivity of those effects in the upright perception of pitch turns.

The perceptual asymmetry of static pitch turns can thus be characterized as follows. In the reclined position, the asymmetrical processing of geometrical cues (Factor 4) between forward and backward turns and the egocentric affordance assessment (Factor 5) were the remaining factors that introduced significant asymmetry. These were impossible to disentangle, and we believe that they are intrinsically related. In the upright position, the former functional adaptation specificity, together with the processing of top-down cues (provided by the scene navigability evaluation), were responsible for the asymmetrical perception of static pitch turns.

\section{GENERAL DISCUSSION}

We have described several asymmetry factors that could influence, at different levels, the perception of yaw and pitch turns: from the processing of motion cues (path integration) or static cues (extraction of geometry) to higher cognitive cues (evaluation of scene navigability). Each of these factors could be related to either the inertial or the visual cues and, thus, could be defined in an earth-centric or an egocentric reference frame, respectively. We analyzed the respective contribution of these factors in three experiments in which two categories of perceptive cues might be available: motion cues provided by the optic flow of the turning simulation and static cues provided by the geometry of the corridor itself.

The first observation concerns the perception of visually presented yaw body turns in the upright position, which corresponds to the natural conditions involved in human locomotion. Visual turn estimates globally undershot the true angle by about $30 \%$ when only motion or static cues were available during the simulation, in comparison with the same turns with both cues. Estimated leftward and rightward turns were, as was expected, perfectly symmetrical across all subjects. In the same natural conditions, a strong asymmetry was found between the perception of forward and downward turns when all the cues were present. The experiments conducted here allowed us to show the following influence of each of the factors on this pitch asymmetry. First, eye movements, such as spontaneous OKN or directed target pursuit, known to be asymmetrical for vertical stimulations, were likely to be responsible for the asymmetrical perception of pitch turns in both the upright and the reclined positions. Indeed, these could take part in the path integration mechanisms, in order to estimate the quantity of turn, through the summing of the slow-phase deviations. Second, the estimation of pitch turns based on the perceived geometry of the corridors was, to some extent, asymmetrical, although this could not have been responsible alone for the strong pitch perceptual asymmetry with all the cues. The strength of this factor as a source of asymmetry decreased with larger angles $\left(90^{\circ}\right.$ and above), probably because of the reduced visual information available for analyzing the geometry of such turns. The two previous factors were defined in an egocentric reference frame and were tilted together with the subjects in the reclined position. Manipulating the body position allowed us to isolate the effect of the gravity-related cognitive cues defined in the earth-centric reference frame from the previous factors. We found a third asymmetry factor that we associated to the distinct evaluation of scene navigability for upward versus downward turns. This includes all top-down influences, such as the fear of falling often reported at the end of the experiments. The last factor was observed in all the experiments, independently of the availability of motion or static cues; in the reclined position, it increased the estimation of turns going in the direction of gravity.

All these factors involved in the asymmetric perception of one's self-rotations can be related to the phylogenetic adaptation of the underlying functional processes to the nominal upright position in the terrestrial gravitational environment.

\section{REFERENCES}

Amorim, M.-A., Glasauer, S., Corpinot, K., \& Berthoz, A. (1997). Updating an object's orientation and location during nonvisual navigation: A comparison between two processing modes. Perception \& Psychophysics, 59, 404-418.

André-Deshays, C., Israël, I., Charade, O., Berthoz, A., Popov, K., \& Lipshits, M. (1993). Gaze control in microgravity: I. Saccades, pursuit, eye-head coordination. Journal of Vestibular Research, 3, 331-343.

Bertin, R. J., IsraëL, I., \& Lappe, M. (2000). Perception of twodimensional, simulated ego-motion trajectories from optic flow. $\mathrm{Vi}$ sion Research, 40, 2951-2971.

Clément, G., Vieville, T., Lestienne, F., \& Berthoz, A. (1986). Modifications of gain asymmetry and beating field of vertical optokinetic nystagmus in microgravity. Neuroscience Letters, 63, 271-274.

Cohen, M. M., \& Larson, C. A. (1974). Human spatial orientation in the pitch dimension. Perception \& Psychophysics, 16, 508-512.

Cutting, J. E., Vishton, P. M., Flǘckiger, M., Baumberger, B., \& GERndT, J. D. (1997). Heading and path information from retinal flow in naturalistic environments. Perception \& Psychophysics, 59, 426-441.

GiBson, J. J. (1979). The ecological approach to visual perception. Boston: Houghton Mifflin.

Grasso, R., Glasauer, S., Takei, Y., \& Berthoz, A. (1996). The pre- 
dictive brain: Anticipatory control of head direction for the steering of locomotion. NeuroReport, 7, 1170-1174.

Grigorova, V. K., \& KorniLOVA, L. N. (1996). Microgravity effect on the vestibulo-ocular reflex is dependent on otolith and vision contributions. Aviation, Space, \& Environmental Medicine, 67, 947-954.

IsRaËL, I., Bronstein, A. M., Kanayama, R., FAldon, M., \& Gresty, M. A. (1996). Visual and vestibular factors influencing vestibular "navigation." Experimental Brain Research, 112, 411-419.

IsRaËL, I., Sievering, D., \& KoenIG, E. (1995). Self-rotation estimate about the vertical axis. Acta Oto-laryngologica, 115, 3-8.

Ivanenko, Y., Grasso, R., IsraëL, I., \& Berthoz, A. (1997). Spatial orientation in humans: Perception of angular whole-body displacements in two-dimensional trajectories. Experimental Brain Research, $117,419-427$

Knierim, J. J., McNaughton, B. L., \& Poe, G. R. (2000). Threedimensional spatial selectivity of hippocampal neurons during space flight. Nature Neuroscience, 3, 209-210.

Lappe, M., Bremmer, F., \& van den Berg, A. V. (1999). Perception of self-motion from visual flow. Trends in Cognitive Sciences, 3, 329336.

LoOMIS, J. M., \& BEALL, A. C. (1998). Visually controlled locomotion: Its dependence on optic flow, three-dimensional space perception, and cognition. Ecological Psychology, 10, 271-285.

Matsuo, V., Cohen, B., Raphan, T., De Jong, V., \& Henn, V. (1979). Asymmetric velocity storage for upward and downward nystagmus. Brain Research, 176, 159-164.

Mittelstaedt, H. (1999). The role of the otoliths in perception of the vertical and in path integration. In B. Cohen \& B. J. M. Hess (Eds.), Otolith function in spatial orientation and movement (Annals of the New York Academy of Sciences, Vol. 871, pp. 334-344). New York: New York Academy of Sciences.

Pozzo, T., Berthoz, A., \& Lefort, L. (1990). Head stabilization during various locomotor tasks in humans: I. Normal subjects. Experimental Brain Research, 82, 97-106.

Stackman, R. W., Tullman, M. L., \& Taube, J. S. (2000). Maintenance of rat head direction cell firing during locomotion in the vertical plane. Journal of Neurophysiology, 83, 393-405.

Taube, J. S., Muller, R. U., \& RanCK, J. B., JR. (1990). Head-direction cells recorded from the postsubiculum in freely moving rats: II. Effects of environmental manipulations. Journal of Neuroscience, 10, 436-447.

Vidal, M., Amorim, M.-A., \& Berthoz, A. (2004). Navigating in a virtual three-dimensional maze: How do egocentric and allocentric reference frames interact? Cognitive Brain Research, 19, 244-258.

VIDAL, M., \& BERTHOZ, A. (2005). Navigating in a virtual 3D maze: Body and gravity, two possible reference frames for perceiving and memorizing. Spatial Cognition \& Computation, 5, 139-161.

WANN, J. P., \& SWAPP, D. K. (2000). Why you should look where you are going. Nature Neuroscience, 3, 647-648.

WARREN, W. H., JR., \& HANNON, D. J. (1988). Direction of self-motion is perceived from optical flow. Nature, 336, 162-163.

Warren, W. H., JR., KaY, B. A., Zosh, W. D., Duchon, A. P., \& Sahuc, S. (2001). Optic flow is used to control human walking. Nature Neuroscience, 4, 213-216.

Wilson, V. J., \& Melvill Jones, G. (1979). Mammalian vestibular physiology. New York: Plenum.

Young, L. R. (1984). Perception of the body in space: Mechanisms. In I. Darian-Smith (Ed.), Handbook of physiology: The nervous system III (pp. 1023-1066). Bethesda, MD: American Physiological Society.

Young, L. R., Oman, C. M., \& Dichgans, J. M. (1975). Influence of head orientation on visually induced pitch and roll sensation. Aviation, Space, \& Environmental Medicine, 46, 264-268.

\section{NOTES}

1. Affordance, as defined by Gibson in 1979, is "a specific combination of the properties of its substance and its surface taken with reference to an animal."

2. Throughout this article, the terms pitch and yaw will refer to rotations described in the egocentric reference frame of the observer. Thus, both a pitch-forward turn for an upright observer and a yaw-rightward turn for an observer reclining on his or her right side will curve downward with respect to gravity.

(Manuscript received June 13, 2005; revision accepted for publication January 30, 2006.) 